\title{
Experience of sport stacking in Korean older adults
}

\author{
Chae-Hee Park* \\ Department of Sport and Healthy Aging , Korea National Sport University, Seoul, Korea
}

Today sedentary lifestyles are a major public health concern and there are many evidences related to physical inactivity. The well-documented evidence is that regular physical activity is an essential part for improving overall health with advancing aging and the most efficient means playing roles of rehabilitation and prehabilitation. However, there are few activity programs which could motivate older adults to continue and maintain their active lifestyles. The purpose of the current study was to describe experience of sport stacking which applied to Korean older adults in the first time. Eighteen older adults living in the community in Seoul which was the capital and largest metropolis of the Republic of Korea participated in the study. A qualitative interview and a survey were conducted and data were analyzed by interpretive content analysis. Indi- vidual, semistructured, face-to-face interviews were recorded, transcribed, condensed and coded to find themes. The main themes were identified regarding older adults' experience of sport stacking: enjoyment of sport stacking, attitudes toward sport stacking as a physical activity program, and benefits from sport stacking. In conclusions, Korean older adults had positive experience of sport stacking and it was necessary for developing strategies to spread it as the physical activity program for older persons in Korea to improve their health and quality of life.

Keywords: Korean older adults, Experiences, Sport stacking, Physical activity

\section{INTRODUCTION}

As the development of medical technology and interest in health grows, a society that dreams of 100 years of age is coming. South Korea is the one of the nations that population aging is the fastest growing in the world. In Korea, the proportion of elderly people aged 65 or older reached $7.2 \%$ in 2000 and it is expected to enter the aged society $(14 \%-21 \%$ of the population are 65 years or older) in 2017, and super aged nation where more than one in five of the population is 65 or older in 2026 (Statistics Korea, 2015).

Along with the progress of population aging trend, various social problems were emerging, such as an increase in elderly people living alone, an increase in the crime of the elderly, and an increase in the suicide rate of the elderly, especially the increase in medical expenses brought a heavy burden on the national finances, each families who support the elderly as well as the elderly people themselves. Therefore, the health of the elderly is an important issue that not only increases happiness and well-being of the elderly themselves, but also reduces the burden on the nation and the people.
Many studies suggest that regular physical activity is a vital element for maintaining health and improving quality of life including physiological, psychological and social aspects in later lives. Physical activity is playing important roles of preventing a diverse chronic disease such as diabetes, cardiovascular disease, cancer, depression, and osteoporosis. This also brings an ability of activities of daily living improvement, elevation of muscular strength, reduction of physical disability, lowering blood pressure, reduction of falls, attenuation of joint pain and lessening of metal disorders (American College of Sports Medicine, 2006; King, 2001).

Despite the proven effectiveness of physical activity, rates of regular physical activity participation in older adults are not high. Approximately 80\% of Korean elderly people aged 60 to 70 are not participating in moderate-intensity physical activity, and most of the Korean elderly aged 71 or older are not participating in continuous physical activity. Most of the domestic elderly exercise programs are aerobic exercise, walking, and stretching. Although these exercise has been shown to have a significant effect on the improvement of muscle strength, flexibility, and balance,

\footnotetext{
${ }^{*}$ Corresponding author: Chae-Hee Park (D) http://orcid.org/0000-0001-8880-3729 Department of Sport and Healthy Aging, Korea National Sport University, 1239 Yangjae, Songpa-gu, Seoul 05541, Korea

Tel: +82-2-410-6953, Fax: +82-2-418-1877, E-mail: chaepark@knsu.ac.kr Received: January 3, 2017 / Accepted: February 8, 2017
} medium, provided the original work is properly cited. 
it is hard to say that older adults like to do them everyday for their own motivation. Therefore, to make physical activity as part of habitual behavior for older adults, it is urgent to supply physical activity programs that enable elderly people to stay safe and interesting.

Sport stacking (cup stacking), began in the early 1980s as a recreational pursuit, is a fairly new sport but it is a popular activity taught in many physical education program in the United States. People use both hands to make a pyramid and then returning the cups into stacks with 12 specialized cups and that must be done in predetermined sequences (Hart and Bixby, 2005). Sport stacking, which can be executed individually or as a team endeavor. The stacking tasks progress from simple structures (three cups; two base cups with one on top) using deliberate, cautious movements to more complicated arrangements (3-6-3) employing quick actions that require highly coordinated, sequential, and accurate movements with both hands involved.

Sport stacking is a sports program that is currently being used by more than 32,000 schools around the world. Many studies suggested benefits of sport stacking for examples, hand-eye coordination (Hart et al., 2006), reaction time (Gibbons et al., 2007; Liggins et al., 2007), bilateral coordination (Rhea et al., 2006), and dualhemispheric brain activity (Hart and Bixby, 2005). Also, Murray et al. (2009) suggested that the metabolic equivalents value for sport stacking is similar to other activities for example, bowling, dance, volleyball, weight lifting which considered as the moderate-intensity exercise. Empirical evidence supporting these claims, however, applying a sport stacking program to older adults is very rare and limited.

Qualitative researches can offer insight into individual perspectives and explore experiences and perceptions that cannot be elicited through quantitative methods (Patton, 2002). Prior to the verification of the effect through the quantitative method of sports stacking for elderly who dislikes the challenge to new things, the experience of elderly people with sports stacking research should be preceded employing the qualitative method. This will allow the elderly to minimize the sense of refusal to cultural or new physical activity programs.

The purpose of this study was to investigate the experiences of the elderly in sport stacking program based on the research question "what are the experiences that elderly people get through sport stacking?"employing the voices of elderly participants in the program. Furthermore, the study was explored the possibility and strategy of whether or not sport stacking culturally and emotionally became effective with the elderly exercise program which could adapt to the emotions of the elderly and could be enjoyed by many older adults in Korea.

\section{MATERIALS AND METHODS}

\section{Subjects}

Participants were a purposeful sample of 18 healthy older adults, aged 68 to 79 years with more than 10 weeks of participating in sport stacking program at senior welfare centers. The respondents lived in Seoul region, all informants provided written informed consent, and only individuals who consent to have their responses audiotaped were included in this part of the study. The study was conducted from October 3, 2015 to April 29, 2016 and the study was approved by Korea National Sport University.

\section{Data collection}

Open-ended semistructured individual interviews were completed and interview questions were related to participation in the program. For example, interviewees were asked about how they had learned about sport stacking program, the nature and extent of their own participation, and their perceptions of the strengths and weaknesses of the program. All interviews were performed face-toface by the author. Each interview, which lasted between $30 \mathrm{~min}$ and $1 \mathrm{hr} 10 \mathrm{~min}$, was recorded, transcribed and then the content analyzed to identify themes relevant to the goals of the study.

In addition, a short survey about sport stacking attitude was conducted including demographic characteristics (age, gender, and education) and perceived benefits of the program. Each item was rated on a 5-point scale that was scored from 1 to 5, with numbers on the scale anchored by the following phrases: very much dislike $(=1)$, somewhat dislike $(=2)$, neutral $(=3)$, somewhat enjoy (=4), very much enjoy $(=5)$. An example item is as follows: "please rate how much you enjoy sport stacking program? Korean older adults participated in sport stacking program is shown in Fig. 1.

\section{Data analysis}

Eighteen participants completed both the interview and the survey. Descriptive analyses were conducted using IBM SPSS ver. 18.0 (IBM Co., Armonk, NY, USA) to describe the participants' demographic information and experiences of sport stacking program. Qualitative data analysis was conducted regularly throughout data collection. After transcriptions were completed, all materials were read and coded by a researcher experienced in qualitative research to identify emerging categories. Data were sorted 


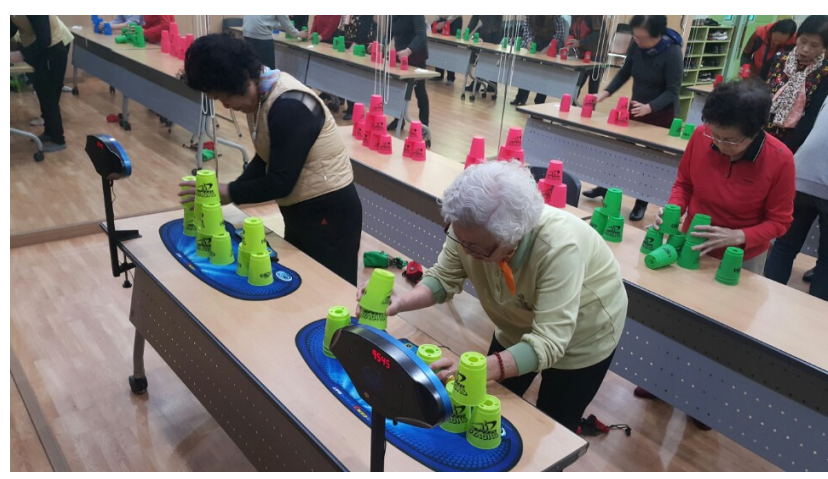

Fig. 1. Sport stacking program with Korean older adults.

Table 1. Baseline characteristics of the informants $(n=18)$

\begin{tabular}{lc}
\hline Characteristic & Number \\
\hline Age (yr) & \\
$65-70$ & 3 \\
$71-75$ & 9 \\
$76-80$ & 6 \\
Gender & \\
Female & 18 \\
Male & 0 \\
Education & \\
No school & 2 \\
Elementary school graduate & 6 \\
Middle school graduate & 6 \\
High school graduate & 3 \\
Bachelor's degree & 1 \\
\hline
\end{tabular}

into themes that describe participants' perceptions and addressed both majority and minority opinions. An inductive approach to analysis was initially employed in order to allow themes to emerge naturally and without imposing assumptions on the data (Patton, 2002).

\section{RESULTS}

A summary of the demographic characteristics of the participants is included in Table 1. The sample ranged in age from 68 to 79 years, with a mean age of 73 . The majority of the sample fell into the 71- to 75-year age category and the participants were all females. Overall, most of the participants graduated from either elementary school or middle school.

Both quantitative and qualitative analyses are presented in relation to three major themes: (a) enjoyment of sport stacking, (b) attitudes toward sport stacking as a physical activity program, and (c) benefits from sport stacking.
Table 2. Overall enjoyment of participating in sport stacking $(n=18)$

\begin{tabular}{lc}
\hline Response & No. $(\%)$ \\
\hline Very much enjoy & $15(83.3)$ \\
Somewhat enjoy & $1(5.6)$ \\
Neutral & $2(11.1)$ \\
Somewhat dislike & $0(0)$ \\
Very much dislike & $0(0)$ \\
\hline
\end{tabular}

\section{Enjoyment of sport stacking}

Table 2 indicated that most of participants liked to participate in sport stacking program and more than $80 \%$ reported they enjoyed sport stacking very much.

The interviews also revealed the participants enjoyed sport stacking because it stimulated curiosity, allowed them to concentrate, and competed with each other to create a sense of challenge. It was also fun to play. Not only did it them give the desire to continue, but also it increased their creativity by acquiring sport stacking as a new activity. The following quotations support the subjects' enjoyment:

It's a new exercise that stimulated curiosity,... it was fun to watch with others as well as enjoying it myself as well. I thought I could focus more and after I went home I still thought of cups and sounds from moving cups. (73-year-old woman)

Another respondent stated,

At first, I could not think of how I could do these cups. It had not been in my head even though I had seen people who were very good at it. Now I became accustomed and focused when I moved cups... after that I like it so much. (77-year-old woman)

The enjoyment from sport stacking motivated them to continue the activity in their everyday lives. Motivation is defined as the forces acting on or within a person to initiate a behavior (Phillips et al., 2004). It seemed to empower participants to become self-directed about sport stacking participation. One participant said, "I thought it would not be fun when I did not know it. However, I enjoyed it so much to do everyday especially I was so proud of myself that I could play it with my granddaughter."

\section{Attitudes toward sport stacking as physical activity program}

Most of participants were aware of importance of regular physical activity for their health. Additionally, they knew very much 
about benefits of physical activity and they perceived sport stacking as one of physical activities. Reasons for engaging in sport stacking were to stay healthy, to avoid further physical decline, and to fight the effects of aging:

I thought this (sport stacking) is physical activity and the program gave me more energy to do things and stay active. (69-yearold woman)

Majority of participant were worried about Alzheimer disease and other dementing illnesses and they strongly believed that a form of physical activity, sport stacking could prevent or delay those diseases:

Whenever I did other physical activity, I forgot about it when it's done. But when I did it (sport stacking), I had to think a lot about each movements and after I went home, those movements stayed in my head... because I used my brain a lot, I do not worry about dementia. (74-year-old woman)

Another interviewee mentioned,

Nowadays, I used hands a lot because of sport stacking. You know what I mean? I had to use left hand and right hand. I felt I got smarter and it seemed to be good for the brain because it was done by hands, $\ldots$ if I continued do this I did not have to worry about a mental illness such as dementia. (66-year-old woman).

\section{Benefits from sport stacking}

A complex interplay of physical, psychological and social factors influenced participation in sport stacking among participants. All participants reported that sport stacking had some benefits and especially they felt that their health had been improved due to the program. Table 3 indicated that over two-thirds of the participants (72.2\%) improved their health including physical, psychological, social health.

Table 3. Health improvement due to sport stacking

\begin{tabular}{lc}
\hline Response & No. (\%) \\
\hline Very much improved & $10(55.5)$ \\
Somewhat improved & $3(16.7)$ \\
Neutral & $3(16.7)$ \\
little improved & $2(11.1)$ \\
Very little improved & $0(0)$ \\
\hline
\end{tabular}

The interview data also showed how participants received benefits through participation in sport stacking. The following examples illustrate a kind of benefits they obtained:

When I started out, I was having problems with upper body strength particularly both hands and lower body and I've noticed a major improvement in that. I felt a lot better and arms and legs were a lot stronger than they were before I started. (76-year-old woman)

Another respondent stated,

I did not do it (sport stacking) well at first, but I was confident that I was getting better, and I actually had symptoms of depression, but it seemed to be getting better. (72-year-old woman)

Another interview yielded the following:

I did not miss sport stacking because it is so nice seeing people and knowing familiar faces. I made friends through the program and it made me more happy and enjoyable. (73-year-old woman)

\section{DISCUSSION}

To knowledge of the author, this was the first investigation that had researched about sport stacking experience to Korean older adults. The key finding of this study was that Korean older adults could accept sport stacking as the physical activity program and they enjoyed a new form of the activity. Furthermore, all participarnts recommended sport stacking to other elderly for their health improvement and they had a strong intention to continously participate in the sport stacking program. This finding represented a departure from findings regarding sport stacking in younger populations.

Geneally, the older adult population dealt with the threat or presence of chronic conditions such as diabetes, heart diseases, and coronary donditions and those conditios make differences in ways that active and inactive older people (Leavy and Aberg, 2010). Joint pain and conditions with sarcopenia caused the reduction of certain physical activities as well. However, the study found out that participants who thought their health condition was not good for participating in any physical activity, they could do sport stacking withtout any barriers. Working sport stacking into the physical activity program of senior wefare centers can result in 
older adults engaging in moderate-intensity physical activity as well as potentially improving their health and fitness components.

Demencia including alzheimer disease are major sources of morbidity and mortality (Larson et al., 2004) that affect millions of people in the increasingly aging society of south Korea. With the prospect of more than 100 million dementia patients in 2050, Korea seems to be the fastest growing country in the world with dementia (Seo, 2016). Many studies demonstrated that modest levels of physical exercise are associated with delayed onset of dementia or Alzheimer disease and people participating at least twice a week in a leisure-time physical activity had 50\% lower odds of dementia compared with sedentary persons (Larson et al., 2006). The study findings may be useful when planing culturally different new physical activity program for health promotion interventions in the older adult population. However, futuer studies should explore relationships between sport stacking and different cronic diseases particularly demencia.

A mix of quantitative and qualitative methods could build an evidence base to understand sport stacking and older adults to develop a new form of community-based physical activity program. Even thought this study provides a starting point for new work, there are some limitations to this study that should be mentioned. First, the participants were limited to a selected population, that is Korean older adults living in urban areas who could participate in a sport stacking study; thus, they may be more positive toward sport stacking and better informed than elderly in general. Second, all participants were Korean women, and the results may not be transferable to men as well as older persons who have different cultural and social backgrounds.

\section{CONFLICT OF INTEREST}

No potential conflict of interest relevant to this article was reported.

\section{ACKNOWLEDGMENTS}

This work was supported by the Research Grant of Korea National Sport University.

\section{REFERENCES}

American College of Sports Medicine. ACSM's guidelines for exercise testing and prescrip-tion. 7th ed. Philadelphia (PA); Lippincott Williams \& Wilkins; 2006.

Gibbons E, Hendrick JL, Bauer JA. Distribution of practice on cup stacking performance. Res Q Exerc Sport 2007;78:A46-47.

Hart MA, Bixby WR. EEG activation patterns during participation in a cup-stacking task. Res Q Exerc Sport 2005;76:A57.

Hart MA, Smith LA, DeChant-Bruennig A. Effect of participation in a cup stacking unit on hand-eye coordination of elementary children. Phys Educ 2006;63:154-159.

King AC. Interventions to promote physical activity by older adults. J Gerontol A Biol Sci Med Sci 2001;56 Spec No 2:36-46.

Larson EB, Shadlen MF, Wang L, McCormick WC, Bowen JD, Teri L, Kukull WA. Survival after initial diagnosis of Alzheimer disease. Ann Intern Med 2004;140:501-509.

Larson EB, Wang L, Bowen JD, McCormick WC, Teri L, Crane P, Kukull $W$. Exercise is as-sociated with reduced risk for incident dementia among persons 65 years of age and older. Ann Intern Med 2006;144: 73-81.

Leavy B, Aberg AC. "Not ready to throw in the towel": perceptions of physical activity held by older adults in Stockholm and Dublin. J Aging Phys Act 2010;18:219-236.

Liggins T, Coleman D, Solis A, Yuhua L. Effects of a cup-stacking exercise program on mo-tor development for school children. Res Q Exerc Sport 2007;78:A47.

Murray SR, Udermann BE, Reineke DM, Battista RA. Energy expenditure of sport stacking. Phys Educ 2009;66:180-185.

Patton MQ. Qualitative research and evaluation methods. 3rd ed. Thousand Oaks (CA): Sage Publications Inc.; 2002.

Phillips EM, Schneider JC, Mercer GR. Motivating elders to initiate and maintain exercise. Arch Phys Med Rehabil 2004;85(7 Suppl 3):S52-57.

Rhea CK, Ludwig K, Mokha M. Changes in upper-limb coordination and kinematics follow-ing a five-week instructional unit on cup stacking. Indiana AHPERD 2006;35:27-28.

Seo YJ. 2050 World dementia patients 100 million and Korea's fastest increase to 271 mil-lion. Joongang Daily. [Internet]. 2016 Jan 27 [cited 2017 Jan 3]. Available from: http://news.joins.com/article/17027521.

Statistics Korea. 2015 elderly statistics [Internet]. Daejeon: Statistics Korea; 2015 [cited 2017 Jan 3]. Available from: http://kostat.go.kr/portal/korea/kor_nw/2/1/index.board?bmode=read\&aSeq=348565. 